\title{
Far-field properties of a line source-excited electrically small quadrupolar metamaterial cylindrical shell
}

\section{Arslanagic, Samel; Ziolkowski, Richard W.; Breinbjerg, Olav}

\section{Published in:}

International Workshop on Antenna Technology: Small and Smart Antennas Metamaterials and Applications, 2007. IWAT '07.

Link to article, DOI:

10.1109/IWAT.2007.370153

Publication date:

2007

Document Version

Publisher's PDF, also known as Version of record

Link back to DTU Orbit

Citation (APA):

Arslanagic, S., Ziolkowski, R. W., \& Breinbjerg, O. (2007). Far-field properties of a line source-excited electrically small quadrupolar metamaterial cylindrical shell. In International Workshop on Antenna Technology: Small and Smart Antennas Metamaterials and Applications, 2007. IWAT '07. (pp. 376-379). IEEE. https://doi.org/10.1109/IWAT.2007.370153

\section{General rights}

Copyright and moral rights for the publications made accessible in the public portal are retained by the authors and/or other copyright owners and it is a condition of accessing publications that users recognise and abide by the legal requirements associated with these rights.

- Users may download and print one copy of any publication from the public portal for the purpose of private study or research.

- You may not further distribute the material or use it for any profit-making activity or commercial gain

- You may freely distribute the URL identifying the publication in the public portal 


\title{
Far-Field Properties of a Line Source-Excited Electrically Small Quadrupolar Metamaterial Cylindrical Shell
}

\author{
Samel Arslanagic ${ }^{(1)}$, R. W. Ziolkowski ${ }^{*}$, and Olav Breinbjerg ${ }^{(1)}$ \\ (1) Orsted $\bullet D T U$, Electromagnetic Systems, Technical University of Denmark, DK-2800 Kgs. Lyngby, Denmark \\ E-mail: sar@oersted.dtu.dk \\ (2) Department of Electrical and Computer Engineering, University of Arizona, Tucson, AZ 85721-0104 \\ E-mail: ziolkowski@arizona.ece.edu
}

\begin{abstract}
An antenna configuration that consists of an arbitrarily located electric line source that radiates in the presence of concentric metamaterial cylinders is examined. With the emphasis on the so-called quadrupolar structure, it is shown that such electrically small configurations may offer significant enhancements of the radiated power. Furthermore, the possibility of controlling the pattern by properly locating the electric line source with respect to the structure, thereby offering a means of obtaining electrically small metamaterial-based directive antennas, is also demonstrated.
\end{abstract}

\section{INTRODUCTION}

Recently, a tremendous interest has emerged in classes of metamaterials (MTMs), such as double-negative (DNG) and single-negative (SNG) materials, and combinations of these with conventional double-positive (DPS) materials. The possibility of designing electrically small MTM-based structures with characteristics that significantly surpass those for structures based on DPS materials have been demonstrated. In particular, highly sub-wavelength resonant structures, such as waveguides, cavities, scatterers and radiators, not realisable with DPS materials alone, have been designed by clever arrangements of DPS, DNG, and/or SNG materials, see e.g., [1]-[4].

In this work, an antenna configuration that consists of an arbitrarily located, infinitely long, electric line source (ELS) which radiates in the presence of infinitely long concentric MTM circular cylinders is analysed. It is shown that electrically small resonant versions of these nested structures can be designed with the procedures outlined in [3], thereby leading to significant enhancement of the radiated power as compared to the power radiated by the ELS in free space. In particular, the quadrupolar resonant structure is examined with respect to its radiated power and its two-dimensional directivity (henceforth referred to as the directivity). The possibility of controlling the directivity for such an electrically small MTM-based structure is likewise demonstrated. This work is an extension of [3], where a detailed treatment of the scattering configurations and figures of merit was given, as well of [4] where near-field and far-field properties of the socalled dipolar structures were investigated. Throughout this manuscript, the time factor $\exp (j \omega t)$, with $\omega$ being the angular frequency, and $t$ being the time, is assumed and suppressed.

\section{CONFIGURATION AND THEORY}

The details of the analytical solution can be found in [3] and only its main points are summarized here. The configuration of interest is depicted in Fig. 1. A cylinder (region 1) with radius $\rho_{1}$ is covered with a cylindrical layer (region 2) of radius $\rho_{2}$, both of which are surrounded by free space (region 3). Regions 1 and 2 are composed of simple and lossy DPS, DNG, and/or SNG materials. The concentric cylinders are illuminated by an infinite ELS of constant electric current $I_{e}$. The ELS is parallel to the common axis of the cylinders and the ELS can be located in any of the three regions. Region $i$, with $i=1,2,3$, is characterized by a permittivity, $\varepsilon_{i}=\varepsilon_{i}^{\prime}-j \varepsilon_{i}^{\prime}$, a permeability, $\mu_{i}=\mu_{i}^{\prime}-j \mu_{i}^{\prime \prime}$, and the wave number $k_{i}=\omega \sqrt{\varepsilon_{i} \mu_{i}}$, where the branch of the square root is chosen such that $\operatorname{Im}\left\{k_{i}\right\} \leq 0$. A cylindrical coordinate system $(\rho, \varphi, z)$ and the associated Cartesian coordinate system $(x, y, z)$ are introduced such that the $z$-axis coincides with the common axis of the cylinders. The coordinates of the observation point and the ELS are $(\rho, \varphi)$ and $\left(\rho_{s}, \varphi_{s}\right)$, respectively. 


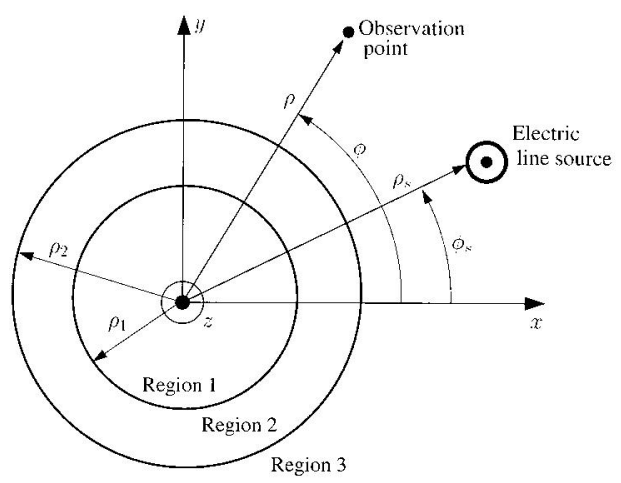

Fig. 1 The configuration - An ELS-excited concentric pair of MTM cylinders.

The solution to the present problem is straightforward, and proceeds as follows. The field generated by the ELS in an infinite medium characterized by $\varepsilon_{i}, \mu_{i}$ and $k_{i}$ is given by the well-known expansion in terms of cylindrical wave functions [5]. In similitude to this field, the unknown scattered field in the region containing the ELS and the unknown total fields in the other regions are also expanded in terms of cylindrical wave functions. These fields involve unknown expansion coefficients: $C_{1 n}, C_{2 n}, C_{3 n}$, and $C_{4 n}$, where $C_{1 n}$ is the expansion coefficient for region $1, C_{2 n}$ and $C_{3 n}$ are those of region 2, while $C_{4 n}$ is the expansion coefficient for region 3. These coefficients, which depend on the location of the ELS, are obtained from the solution of the system of equations that result from the enforcement of the boundary conditions, and thus also depend on the values of the cylindrical waves at the two interfaces.

In [3], specific attention was devoted to the so-called power ratio (PR), which is the ratio of the total power radiated by the ELS in the presence of MTM cylinders to the power radiated by the ELS in free space. From the cylindrical wave expansion this can be expressed as

$$
\mathrm{PR}=\frac{1}{2} \sum_{n=0}^{N_{\max }} \varepsilon_{n}^{2}\left(3-\varepsilon_{n}\right)\left|\alpha_{n}\right|^{2}
$$

Based on (1), large PR values were found in [3] for specific MTM-based structures. Presently, the analysis of these structures is continued in terms of their directivity, $D$, which can be expressed as

$$
D(\varphi)=2\left|\sum_{n=0}^{N_{\max }} \varepsilon_{n} j^{n} \alpha_{n} \cos \left[n\left(\varphi-\varphi_{s}\right)\right]\right|^{2} / \sum_{n=0}^{N_{\max }} \varepsilon_{n}^{2}\left(3-\varepsilon_{n}\right)\left|\alpha_{n}\right|^{2}
$$

In (1) and (2), the quantity $\alpha_{n}=J_{n}\left(k_{3} \rho_{s}\right)+C_{4 n}$ when the ELS is in region 3, while $\alpha_{n}=C_{4 n}$ when the ELS is in any other region. Furthermore, the symbol $\varepsilon_{n}$ is the Neumann number, i.e., $\varepsilon_{n}=1$ for $n=0$, and $\varepsilon_{n}=2$ otherwise, while $N_{\max }$ is the truncation limit and is chosen to ensure convergence of the cylindrical wave expansions.

\section{RESULTS AND DISCUSSION}

While a thorough examination of the PR and the directivity for specific resonant dipolar MTM-based structures (i.e., those for which the dominant mode of radiation corresponds to the $n=1$ term) was conducted in [3] and [4], the present work elucidates the properties of lossless and lossy quadrupolar structures (i.e., those for which the dominant mode of radiation corresponds to the $n=2$ term). Only DPS $\left(\varepsilon_{i}^{\prime}>0, \mu_{i}^{\prime}>0\right)$ and DNG $\left(\varepsilon_{i}^{\prime}<0, \mu_{i}^{\prime}<0\right)$ materials are emphasized, and a given structure is referred to by its properties in region 1 and 2. For example, a DPS-DNG structure indicates that region 1 (2) contains a DPS (DNG) material. A case with no loss and three cases where the influence of loss is taken into account are investigated. In the lossless case, Case 1 , it was shown that for a frequency of operation $f_{0}=300 \mathrm{MHz}$ and the lossless 
material parameters of various regions selected as $\varepsilon_{1}=\varepsilon_{3}=\varepsilon_{0}, \varepsilon_{2}= \pm \varepsilon_{0}$ and $\mu_{1}=\mu_{3}=\mu_{0}, \mu_{2}= \pm 4 \mu_{0}$, with $\varepsilon_{0}$ and $\mu_{0}$ denoting the free-space permittivity and permeability, the geometrical parameters for the resonant quadrupolar structure are $\rho_{1}=3.8729 \mathrm{~mm}$ and $\rho_{2}=5.000068 \mathrm{~mm}$ [3]. The negative (positive) parameters in region 2 apply for a DNG (DPS) material. Thus, the specific antenna configurations under investigation are a DNG or a DPS shell in the presence of an arbitrarily located ELS.

Fig. 2(a) shows the PR as a function of the ELS location, $\rho_{s}$, in region 2. While no enhancements are found for the DPS-DPS structure, very large PRs are found for the electrically small DPS-DNG structure when the ELS is located very close to the DNG shell surfaces. As Fig. 2 shows, the PR changes significantly as the location of the ELS is moved through the DNG shell. In particular, no enhancement is found for $\rho_{s}=4.400486 \mathrm{~mm}$. Fig. 2(b) shows the directivity for a number of ELS locations for the DPS-DNG structure. While the directivity has a symmetric quadrupolar form when the ELS is close to the DNG surfaces, it has a symmetric dipolar form with the main lobes along either the $y$ - or $x$-axes, when the ELS is just to the left (e.g., at $\rho_{s}=4.3999 \mathrm{~mm}$ ) and right (e.g., at $\rho_{s}=4.401 \mathrm{~mm}$ ) of $\rho_{s}=4.400486 \mathrm{~mm}$, at which position a clear monopolar pattern is found. As demonstrated in [3] there are no enhancements of the radiated power for the monopolar ( $n=0)$ mode, i.e., an enhanced PR requires the excitation of a higher order mode. This explains the null in the PR as shown in Fig. 2(a).

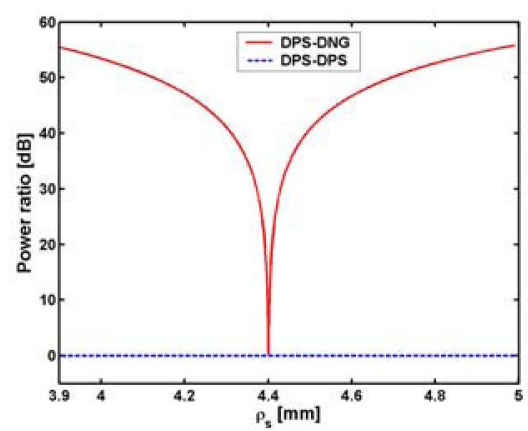

(a)

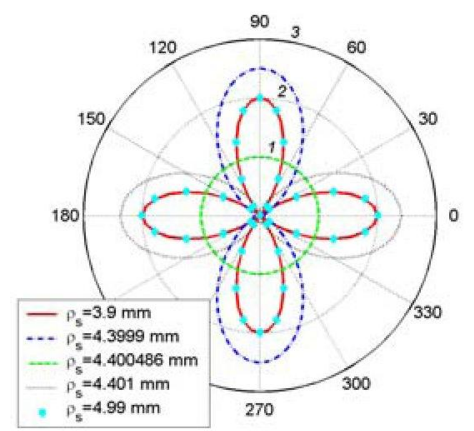

(b)

Fig. 2 Lossless (Case 1) quadrupolar structures - ELS in region 2: (a) PR as a function of the ELS location for DPSDPS and DPS-DNG structures. (b) Directivity patterns for the DPS-DNG structure.

These pattern results demonstrate that it is possible to achieve a gradual transformation of the directivity from the quadrupolar via the dipolar to the monopolar form, and back again, as the ELS is moved through the DNG shell. The details of this transformation are given in Fig. 3, where the directivity is shown for ELS locations near $\rho_{s}=4.3999 \mathrm{~mm}$, $\rho_{s}=4.400486 \mathrm{~mm}$, and $\rho_{s}=4.401 \mathrm{~mm}$. The pattern variations shown in Fig. 3 are in agreement with the PR values reported in Fig. 2(a), and serve as a confirmation of the excitation of a particular resonant mode required for the enhancements of the radiated power. It is important to emphasize that not only can one control the modal form of the of the directivity pattern with the DPS-DNG quadrupolar structure, but also the direction of the enhancement. The change between the vertical and horizontal dipolar patterns as the ELS attains specific locations confirms this behavior.

In the lossy cases regions 1 and 3 resemble those of the lossless case, while in region 2 the following three cases are selected:

- Case 2: $\varepsilon_{2}=\varepsilon_{0}(-1-j 0.0001), \quad \mu_{2}=\mu_{0}(-4-j 0.0001)$;

- Case 3: $\varepsilon_{2}=\varepsilon_{0}(-1-j 0.001), \quad \mu_{2}=\mu_{0}(-4-j 0.001)$;

- Case 4: $\varepsilon_{2}=\varepsilon_{0}(-1-j 0.01), \quad \mu_{2}=\mu_{0}(-4-j 0.01)$.

Thus, the electric and magnetic loss tangents, defined by $L T_{e}=\varepsilon_{i}^{\prime \prime} /\left|\varepsilon_{i}^{\prime}\right|$ and $L T_{m}=\mu_{i}^{\prime \prime} /\left|\mu_{i}^{\prime}\right|$, are $L T_{e}=0.0001, L T_{m}=0.000025$ (Case 2); $L T_{e}=0.001, L T_{m}=0.00025$ (Case 3); and $L T_{e}=0.01, L T_{m}=0.0025$ (Case 4). Although both electric and magnetic losses are included, one must note that it is the magnetic loss that is crucial when the 
source of radiation is an ELS since it is the permeability, and not the permittivity, of the DNG shell that determines the overall behaviour of the structure. As illustrated in Fig. 4(a), the PR curve is now broadened and the radiated power enhancements are diminished as the losses are increased. Even a small amount of loss (Case 2) has a profound impact on the PR. Nonetheless, it is only after the inclusion of even more loss (Cases 3 and 4) that practically no enhancement occurs. The inclusion of loss gradually suppresses the excitation of the resonant higher order modes at the cost of the monopolar mode. This is confirmed by the directivity results given in Fig. 4(b). While Case 2 has the same directivity as in the lossless case, Case 3 is a mixture of several modes, and Case 4 actually produces a monopolar form of the directivity.

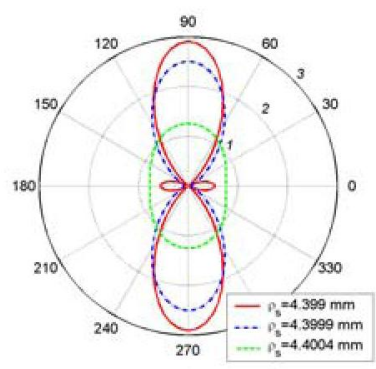

(a)

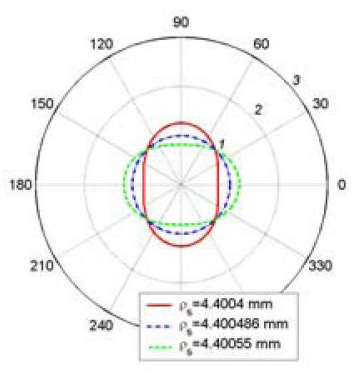

(b)

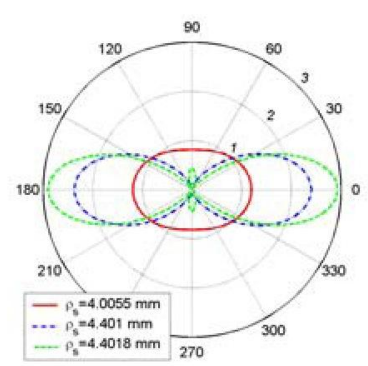

(c)

Fig. 3 The change in the directivity as the ELS location varies in region 2 for the lossless (Case 1) DPS-DNG structure: to the left (a), near and at (b), and to the right (c) of the critical radius $\rho_{s}=4.400486 \mathrm{~mm}$.

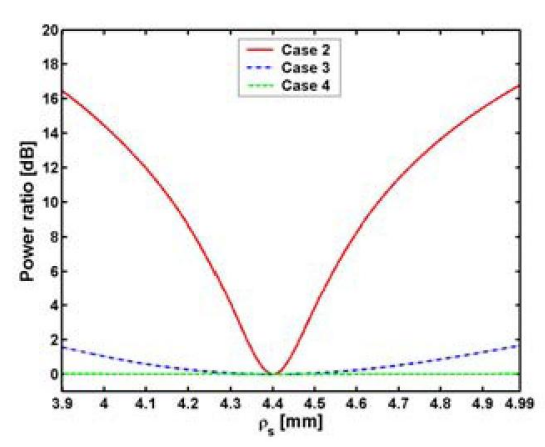

(a)

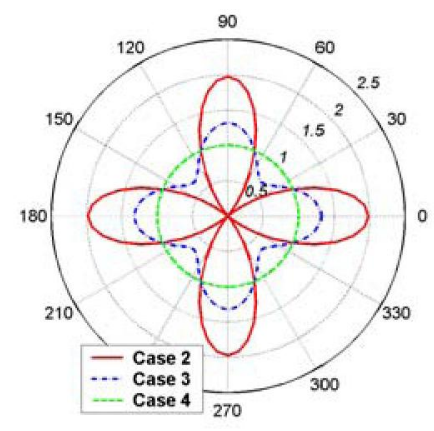

(b)

Fig. 4 Influence of the losses on the directivity for the DPS-DNG structure: Cases 2-4.

\section{REFERENCES}

[1] N. Engheta, and R. W. Ziolkowski, "A positive future for double-negative metamaterials," IEEE Microwave Theory Tech., vol. 53, pp. 1535-1556, Apr. 2005.

[2] A. Alù, and N. Engheta, "Polarizabilities and effective parameters for collections of spherical nano-particles formed by pairs of concentric double-negative (DNG), single-negative (SNG) and/or double-positive (DPS) metamaterial layers," J. Appl. Phys., vol. 97, 094310, May, 2005.

[3] S. Arslanagic, R.W. Ziolkowski, and O. Breinbjerg, "Excitation of an electrically small metamaterial-coated cylinder by an arbitrarily located line source," Microwave Opt. Technol. Lett., vol. 48, pp. 2598-2605, Dec. 2006.

[4] S. Arslanagic, R.W. Ziolkowski, and O. Breinbjerg, "Near-field distribution, directivity and differential scattering cross section for a line source-excited metamaterial-coated electrically small cylinder," EuCap, France, Nov. 2006.

[5] C. A. Balanis, Advanced Engineering Electromagnetics, $2^{\text {nd }}$ ed., John Wiley \& Sons, 1989. 